\title{
Dynamics and Linear Stability Analysis in Semiconductor Lasers with Phase-Conjugate Feedback
}

\author{
Atsushi Murakami and Junji Ohtsubo, Member, IEEE
}

\begin{abstract}
Dynamics of semiconductor lasers with phaseconjugate feedback are studied. At a certain feedback level, the laser output evolves into periodic oscillations due to the undamped relaxation oscillation which corresponds to the highest mode of the linear stability analysis. With increasing feedback level, the second mode is excited in quasi-periodic oscillations. Though the second mode frequency in the conventional feedback is almost the same as the external cavity mode frequency, the frequency in the phase-conjugate feedback is completely different from that. We study the dynamics of the phaseconjugate feedback, showing the time series, power spectrum, and attractors, and compare the results with those in the conventional feedback. The transition of the linear modes is also studied from the linear stability analysis. The results derived from the linear stability analysis and the mode transition are compatible with those of the simulations based on the rate equations.
\end{abstract}

Index Terms - Chaos, conventional feedback, linear stability analysis, phase conjugate feedback, semiconductor laser.

\section{INTRODUCTION}

$\mathbf{S}$ EMICONDUCTOR lasers with optical feedback have been paid much attention as one of the most interesting nonlinear systems to generate optical chaos [1]-[15]. The laser and external cavities form a compound cavity in the optical feedback system. When the light reflected from an external reflector couples into the laser cavity, the laser output is affected considerably. The output power of the compound cavity semiconductor laser shows a rich variety of dynamical behaviors with the change of the parameters which include the reflectivity of the external mirror, the length of the external cavity, and the injection current. The dynamics of the semiconductor laser with optical feedback have been investigated theoretically by analyzing the rate equations together with the feedback terms [1], and the dependence of the chaotic dynamics on the system parameters has been studied in detail. The key to the chaos dynamics is the relaxation oscillation of the laser which remains undamped due to the effect of the external optical feedback [9]-[11]. With nonvanishing relaxation oscillation, the laser output power behaves like periodic oscillation and evolves into quasiperiodic and, finally, chaotic oscillations with the increase of the feedback level [12]-[14]. The stability and instability

Manuscript received April 10, 1998; revised June 29, 1998.

The authors are with the Faculty of Engineering, Shizuoka University, Hamamatsu 432, Japan.

Publisher Item Identifier S 0018-9197(98)07167-X. of semiconductor lasers with optical feedback are important issues not only from the physical aspects but also from the application standpoints of view. In the applications of chaos in external-cavity semiconductor lasers, the chaos control algorithm can be used to suppress the noise which is caused by the optical feedback [3]-[8].

Recently, considerable attention has also been paid to the dynamics of the optical feedback caused by a phase-conjugate mirror [16]-[22]. The research of the phase conjugate feedback done to date has been primarily theoretical. The time response of a phase-conjugate mirror is usually ignored in the theoretical studies. However, an actual phase-conjugate mirror has a finite time response. For this reason, phase-conjugate feedback in semiconductor lasers with a finite-penetration-depth phaseconjugate mirror has also been studied [21]. For a fast-response phase-conjugate mirror such as the nonlinear Kerr medium, the effect of the finite time response may be neglected and the theoretical treatment done previously can be applied to investigate the dynamics compatible with those in the experiments. The dynamics of semiconductor lasers with phase-conjugate feedback are much different from those of conventional optical feedback because the phase of light reflected by a phaseconjugate mirror is reversed and the phase-conjugate light is coupled into the laser cavity. Although the laser output power also behaves like stable, periodic, and chaotic oscillations with the variations of the parameter values, the laser output of the phase-conjugate feedback behaves in a rather unstable manner compared with those of the conventional feedback. For example, the stable regions of the laser output power for both models have similar periodic structures as a function of the external cavity length, however, stable peak locations for each model are completely out of phase [22]. In spite of several investigations of phase-conjugate feedback [16]-[20], the dynamics are still not clarified and a few studies have been done for comparison between the dynamics of the phaseconjugate and conventional feedback.

In this paper, a study of the dynamics of semiconductor lasers with phase-conjugate feedback is conducted. We are concerned with the relaxation oscillation and the external mode oscillations due to the external mirror to investigate the dynamics and chaotic behaviors. It is shown that the second mode frequency excited by the external mirror in phaseconjugate feedback is completely different from the frequency corresponding to the external cavity length or the frequency excited in conventional feedback. In order to characterize the 
dynamics of phase-conjugate feedback, three-dimensional (3D) attractors are also employed. These dynamics are studied by numerically calculating the rate equations. On the other hand, the linear stability analysis is also conducted for phaseconjugate feedback and the dynamics are explained based on the analysis. The distribution and transition of the linear modes are investigated by the linear stability analysis and the results are compared with those obtained by the numerical simulations based on the rate equations. The stability dependence on the external mirror position and the frequency jump (equivalently laser output power jump) due to the injection current change are well understood by the linear stability analysis.

\section{THEORETICAL BACKGROUND}

Semiconductor lasers with optical feedback are well described by the rate equations based on the Lang and Kobayashi model. The model can be applied not only for the case of conventional optical feedback (CFB) but also for that of phaseconjugate feedback (PCF) [1], [22]. We here briefly summarize the theoretical background of semiconductor lasers with optical feedback. We assume that a phase-conjugate mirror responds instantaneously, for example, such as for a fourwave mixing phase-conjugate reflector with a fast nonlinear medium, and the laser oscillates in a single longitudinal mode with an angular frequency $\omega_{0}$. The complex electric field in the laser active region is written as $E(t) \exp \left[i\left(\omega_{0} t+(\phi(t))\right]\right.$, where $\phi(t)$ is the phase change due to the feedback effect. Then, the rate equations for the amplitude and phase of the complex electric field and the carrier density are given by

$$
\begin{aligned}
\frac{d E(t)}{d t}= & \frac{1}{2}\left\{g\left(N(t)-N_{0}\right)-\frac{1}{\tau_{p}}\right\} E(t) \\
& +\frac{\kappa}{\tau_{\text {in }}} E(t-\tau) \cos \theta(t) \\
\frac{d \phi(t)}{d t}= & \frac{\alpha}{2}\left\{g\left(N(t)-N_{0}\right)-\frac{1}{\tau_{p}}\right\}-\frac{\kappa}{\tau_{\text {in }}} \frac{E(t-\tau)}{E(t)} \sin \theta(t)
\end{aligned}
$$$$
\frac{d N(t)}{d t}=J-\frac{N(t)}{\tau_{s}}-g\left\{N(t)-N_{0}\right\}|E(t)|^{2}
$$

where $g$ is the linear gain coefficient, $N_{0}$ is the carrier density at transparency, $\alpha$ is the linewidth enhancement factor, $J$ is the injection current density, $\tau_{p}$ is the photon lifetime, $\tau_{s}$ is the carrier lifetime, $\tau=2 L / c$ is the external cavity roundtrip time where $L$ is the distance from the laser facet to the external reflector and $c$ is the light velocity in vacuum, and $\tau_{\text {in }}=2 l / c$ is the round-trip time within the laser cavity where $l$ is the internal cavity length and $\eta$ is the refractive index of the laser cavity. $\theta(t)$ represents the phase coupling between the original light in the cavity and the delayed light from the external reflector and is given by the following forms for phase-conjugate and conventional feedback:

$$
\theta_{\mathrm{PCF}}(t)=\phi_{\mathrm{PCF}}+\phi(t)+\phi(t-\tau)
$$

and

$$
\theta_{\mathrm{CFB}}(t)=\omega_{0} \tau+\phi(t)-\phi(t-\tau)
$$

respectively, where $\phi_{\mathrm{PCF}}$ is the constant phase change from the phase-conjugate mirror and this value is chosen to be
$\phi_{\mathrm{PCF}}=0$ without loss of generality. The feedback parameter $\kappa$ is written as $\kappa=\left(1-r_{0}^{2}\right) r / r_{0}$, where $r_{0}$ and $r$ are the amplitude reflectivities of the laser exit facet and the external reflector, respectively. Since multiple reflections between the laser facet and the external reflector are neglected, (1)-(5) are valid for a weak to moderate feedback level. Choosing appropriate values of the injection current $J$, the external reflectivity $r$, and the external cavity length $L,(1)-(5)$ are numerically solved by using a fourth-order Runge-Kutta algorithm. In the numerical simulations, we use the same parameter values listed in [22, Table I].

\section{Dynamics of Phase-Conjugate FeEdBACK}

With increasing the feedback light level, a stable laser output power switches to a periodic state and evolves into quasiperiodic and, finally, chaotic oscillations. The process is more or less the same both for the phase-conjugate and conventional optical feedback cases. We have already shown the route to chaos which is dependent on the system parameters such as the injection current $J$, the external cavity length $L$, and the reflectivity of the external reflector $r$ in [22]. In the case of the basic bifurcations of the laser output power for the increase of the feedback level, two types of the bifurcations have been observed, i.e., the period doubling and quasiperiodic route to chaos. In the following, we will show the numerical studies for the dynamics of the bifurcation process based on the rate equations.

The key to chaos in the dynamics is the relaxation oscillation which becomes undamped due to the presence of the external feedback. In a periodic oscillation, the laser output power has a frequency which is close to the relaxation oscillation frequency of the solitary laser but not always exactly the same as it. Fig. 1 shows the time series and its spectrum of the output power for a periodic state (period-1) at a certain value of the external reflectivity. Fig. 1(a) and (b) is for PCF and Fig. 1(c) and (d) is for CFB. The injection current and the external cavity length are $J=1.5 J_{\mathrm{th}}$ and $L=21.0 \mathrm{~cm}$. The fundamental frequencies of these periodic oscillations are 3.05 (PCF) and $3.24 \mathrm{GHz}$ (CFB), respectively. It is noted that the frequencies are slightly different from each other. Since the relaxation oscillation frequency of the solitary laser is calculated to be $f_{R}=3.25 \mathrm{GHz}$, the frequency of $\mathrm{CFB}$ is almost equal to that of the solitary laser but the frequency of PCF is much different from it.

With increase of the feedback level, the output power shows quasi-periodic oscillation and the second mode frequency beside the relaxation oscillation is excited, as shown in Fig. 2. Fig. 2 shows the time series and its spectrum for quasi-periodic states of PCF and CFB. The values of the excited second mode frequencies (the lowest frequencies in the figure) are 0.235 and $0.567 \mathrm{GHz}$ for $\mathrm{PCF}$ and $\mathrm{CFB}$, respectively, and the corresponding lengths are 63.8 and $26.5 \mathrm{~cm}$. The excited frequency is originated from the external cavity mode and the output power spectrum includes not only this frequency but also their harmonic and entangled frequencies with the relaxation oscillation. In the case where the ratio between the relaxation oscillation and second mode frequencies is rational, 


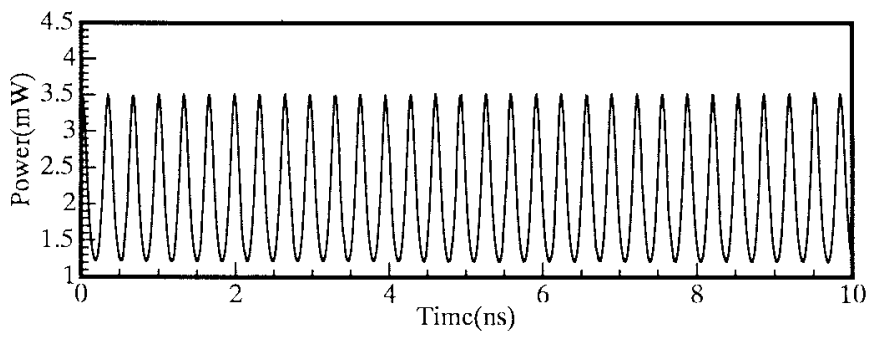

(a)

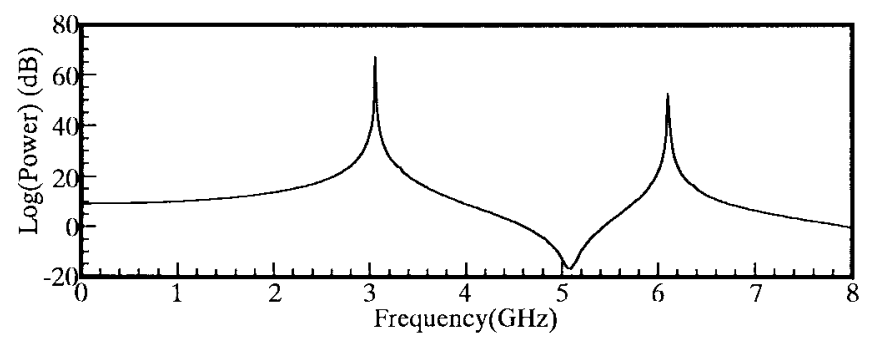

(b)

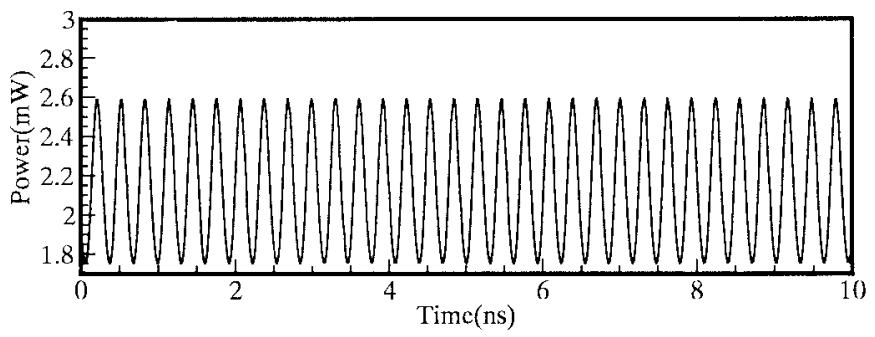

(c)

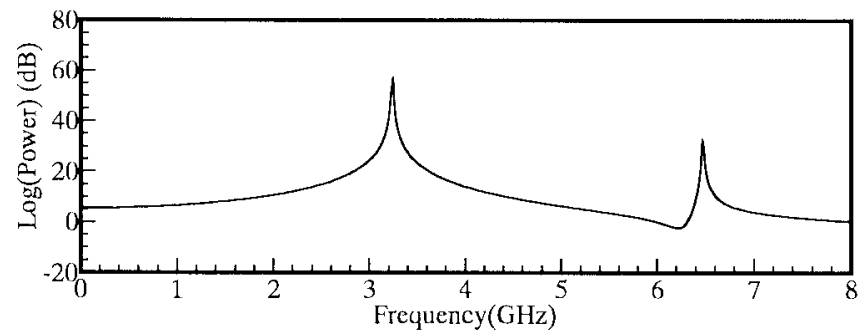

(d)

Fig. 1. Time series of period-1 oscillation and its spectrum at $J=1.5 J_{\text {th }}$ and $L=21.0 \mathrm{~cm}$. (a), (b) PCF at $r=0.45 \%$. (c), (d) CFB at $r=0.3 \%$.

the trajectory in the phase space shows a periodic structure, while the trajectory becomes quasi-periodic for the ratio being irrational. With further increase of the feedback level, the laser output finally evolves into a chaotic state. In general, the second mode frequency for CFB is nominally the external cavity frequency, though the value is slightly smaller than the exact external cavity frequency [23].

As is shown in Fig. 2, the value of the second mode frequency for PCF is much less than that for CFB (equivalently, the corresponding length for $\mathrm{PCF}$ is much larger than that for CFB). We show the relation between the length corresponding to the second mode frequency and the external cavity length $L$ in Fig. 3. The broken line which shows undulation indicates the length corresponding to the second mode frequency for PCF. The valleys of the undulation correspond to the enhanced stability peaks which will be discussed later in Fig. 6. On

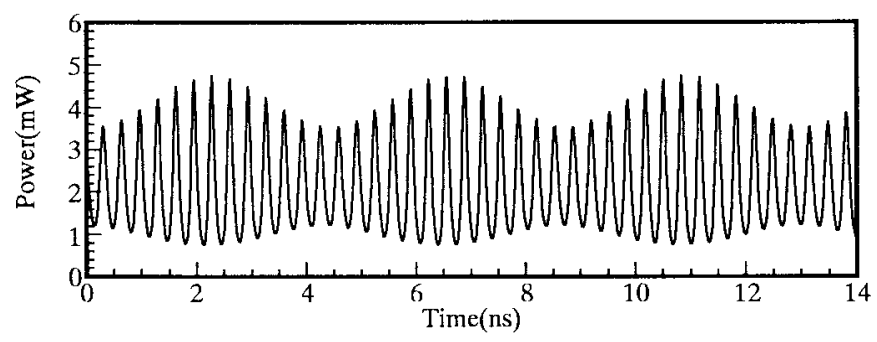

(a)

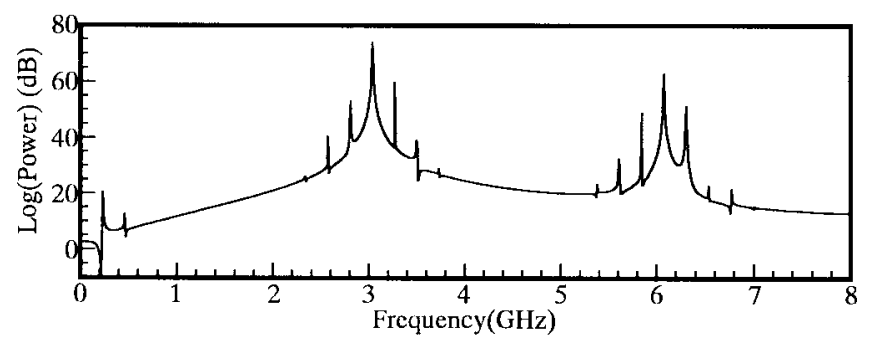

(b)

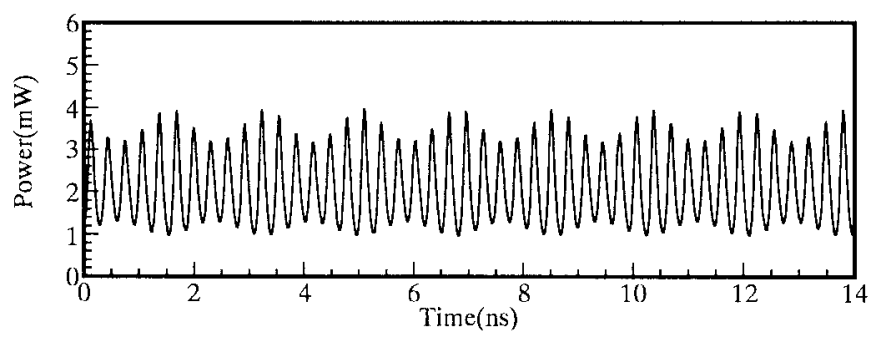

(c)

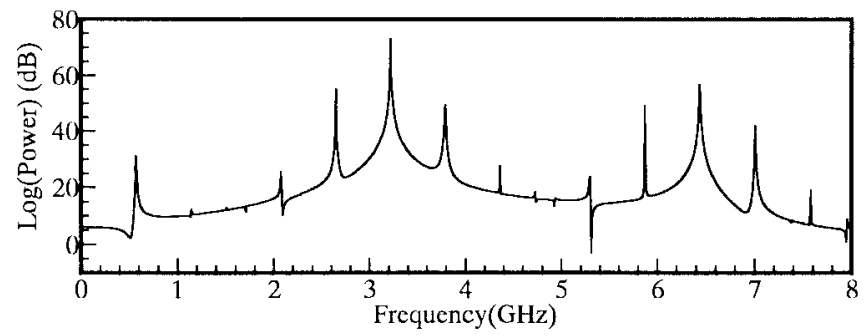

(d)

Fig. 2. Time series of quasi-periodic oscillation and its spectrum at $J=1.5 J_{\text {th }}$ and $L=21.0 \mathrm{~cm}$. (a), (b) PCF at $r=0.52 \%$. (c), (d) $\mathrm{CFB}$ at $r=0.67 \%$

the other hand, the dotted line represents the relation for CFB calculated from the rate equations. The plot for the CFB model has the linear relation, though the value of the length for the second mode frequency is slightly larger than the external cavity length. The black and white circles are the results derived from the linear stability mode analysis which will be discussed later on. The behaviors of the second mode frequency between the PCF and CFB models are much different.

The 3-D reconstruction of attractors is a useful tool to understand the dynamics of nonlinear systems. Fig. 4 shows 3 -D plots of attractors for time-delayed laser output powers $I(t), I(t+\tau)$, and $I(t+2 \tau)$, where $\tau$ is the delay due to the external round-trip time. Fig. 4(a) shows typical examples of attractors for PCF at $J=1.5 J_{\mathrm{th}}$. The upper, middle, 


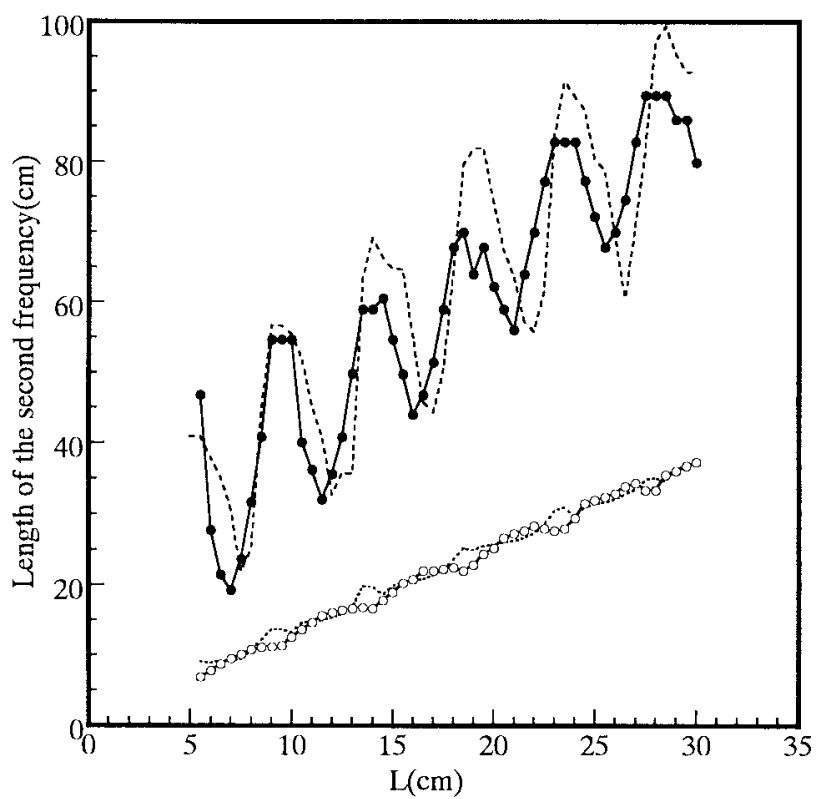

Fig. 3. Plot of the length corresponding to the excited frequency of the second mode against the external cavity length $L$ calculated from the rate equations. Broken line: PCF; dotted line: CFB. Black and white circles are the results obtained from the linear stability analysis, which is discussed in Section IV.

and bottom rows are for period-1, quasi-periodic, and chaotic states, respectively. For the sake of the plot, the scales for period-1 and quasi-periodic states are normalized by each maximum value of the output power variations, while that for chaotic states represents the actual scale of the laser output power. In the plot of attractors for period-1 states, the 2-D projections of the attractors are displayed for reference. The notable feature is that the powers in period-1 state at the time delay $\tau$ almost oscillate in phase as shown in the attractor of $L=9.25 \mathrm{~cm}$. The position of the external mirror corresponds to the stability minimum which will be discussed later in Fig. 6. Furthermore, the volume of the chaotic attractor in this position is compact compared with the others. The left column corresponds to the position of the external mirror for an enhanced stability peak which will also be discussed in Fig. 6. At this position, the laser output power stays stable up to rather high external reflectivity, while the laser output power shows large fluctuations once the state evolves into unstable oscillations. The middle column shows attractors at the position of the external mirror for in-between positions of the stability peak and bottom. For comparison, Fig. 4(b) shows examples of attractors for CFB. In contrast to the case of PCF, the external mirror position of $L=7.00 \mathrm{~cm}$ corresponds to stability minimum, while that of $L=9.25 \mathrm{~cm}$ corresponds to the stability peak. At the stability minimum, the oscillations between $\tau$-delayed output powers for period-1 state are almost out of phase, as shown in the upper left column in Fig. 4(b). Furthermore, the volume of the attractor for CFB is much smaller than that for PCF. This means the fact that the laser output for CFB is much more stable compared with that for PCF. The stability of the laser output depends on the excitation of the relaxation oscillation, as is discussed later on. In the PCF and CFB configurations, the competition between

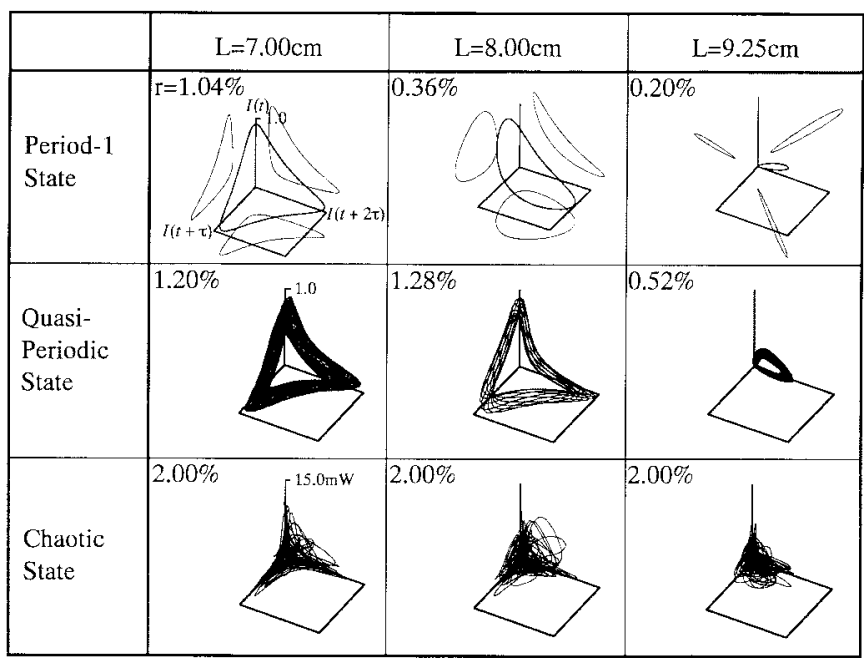

(a)



(b)

Fig. 4. Three-dimensional plot of attractors for $I(t), I(t+\tau)$, and $I(t+2 \tau)$ at $J=1.5 J_{\mathrm{th}}$. (a) Attractors for PCF. Left and right columns correspond to stability maximum and minimum, respectively. (b) Attractors for CFB. Left and right columns correspond to stability minimum and maximum, respectively.

the highest and second highest linear modes suppresses the instability of the laser output at stability peaks. The stable range of the external reflectivity is wide due to linear mode competition, but the laser output soon evolves into unstable oscillation for the increase of the external reflectivity once the state becomes periodic oscillation [22]. On the other hand, the instability is enhanced for a small external reflectivity due to the excitation of only a single relaxation oscillation mode at the stability minima. At the stability minima, the laser output gradually evolves into unstable oscillation like period doubling bifurcations, since the dynamics are related to only a single linear mode.

\section{LiNEAR StABILITY ANALYSIS}

The laser dynamics discussed in the previous section can be also explained by the linear stability analysis for the rate equations. Furthermore, some new interpretation for the 
physical properties can be obtained from the analysis. This section concerns the dynamic behaviors of the laser output power based on such an analysis. We give some results and compare them with those derived from the direct numerical simulations by the rate equations. At first, we briefly summarize the results of the linear stability analysis for (1)-(5). By the linear stability analysis around the stationary solutions for the field amplitude, the phase, and the carrier density, the characteristic equations for the stability in the phase-conjugate and conventional feedback models are given by [2], [22]

$$
\begin{aligned}
D_{\mathrm{DCF}}(\gamma)= & \gamma^{3}+\gamma^{2}\left(\frac{1}{\tau_{R}}+\frac{2 \kappa}{\tau_{\text {in }}} \cos 2 \phi_{s}\right) \\
& +\gamma\left[\omega_{R}^{2}+\frac{2 \kappa}{\tau_{R}} \cos 2 \phi_{s}+A B\left(\frac{\kappa}{\tau_{\text {in }}}\right)^{2}\right] \\
& +\left[\frac{A B}{\tau_{R}}\left(\frac{\kappa}{\tau_{\text {in }}}\right)^{2}+\omega_{R}^{2} \frac{\kappa B}{\tau_{\text {in }}}\right. \\
& \left.\cdot\left(\cos 2 \phi_{s}-\alpha \sin 2 \phi_{s}\right)\right] \\
= & 0
\end{aligned}
$$

and

$$
\begin{aligned}
D_{\mathrm{CFB}}(\gamma)= & \gamma^{3}+\gamma^{2}\left[\frac{1}{\tau_{R}}+\frac{2 \kappa A}{\tau_{\text {in }}} \cos \left(\omega_{s} \tau\right)\right] \\
& +\gamma\left[\omega_{R}^{2}+\frac{2 \kappa A}{\tau_{R} \tau_{\text {in }}} \cos \left(\omega_{s} \tau\right)+\left(\frac{\kappa A}{\tau_{\text {in }}}\right)^{2}\right] \\
& +\left\{\frac{1}{\tau_{R}}\left(\frac{\kappa A}{\tau_{\text {in }}}\right)^{2}+\omega_{R}^{2} \frac{\kappa A}{\tau_{\text {in }}}\right. \\
& \left.\cdot\left[\cos \left(\omega_{s} \tau\right)-\alpha \sin \left(\omega_{s} \tau\right)\right]\right\} \\
= & 0
\end{aligned}
$$

respectively. In above equations, $\phi_{s}$ is the stationary phase of the phase-conjugate feedback, $\omega_{s}$ is the stationary solution for the angular oscillation frequency for $\mathrm{CFB}, A=1-$ $\exp (\gamma t), B=1+\exp (\gamma t)$, and $\tau_{R}^{-1}=\tau_{s}^{-1}+g E_{s}^{2}$, where $\omega_{R}=2 \pi f_{R}\left(\omega_{R}^{2}=g E_{s}^{2} / \tau_{p}\right)$ is the relaxation oscillation angular frequency of the solitary laser and $E_{s}$ is the stationary field amplitude. The above equations have many solutions and they are complex. The solution $\gamma$ obtained from the characteristic equation is referred to as a linear mode. The real part of the solution denotes the stability of the mode and the imaginary part represents the resonance frequency of the mode. The stability and instability of the dynamics in the system can be discussed from the solutions of the linear mode.

The dynamics of the system are studied based on the transition of the linear modes obtained from (6) and (7) with the variations for the external reflectivity. Fig. 5 shows the distributions of linear modes within the range of $0-5$ $\mathrm{GHz}$ at $J=1.3 J_{\mathrm{th}}$ and $L=10.0 \mathrm{~cm}$ calculated from the characteristic equations. In the figure, the vertical and horizontal axes represent the real and imaginary parts of $\gamma$, respectively. Without feedback in Fig. 5(a), i.e., for the case of the solitary laser, there exists only a single linear mode, which corresponds to the laser relaxation oscillation. Since

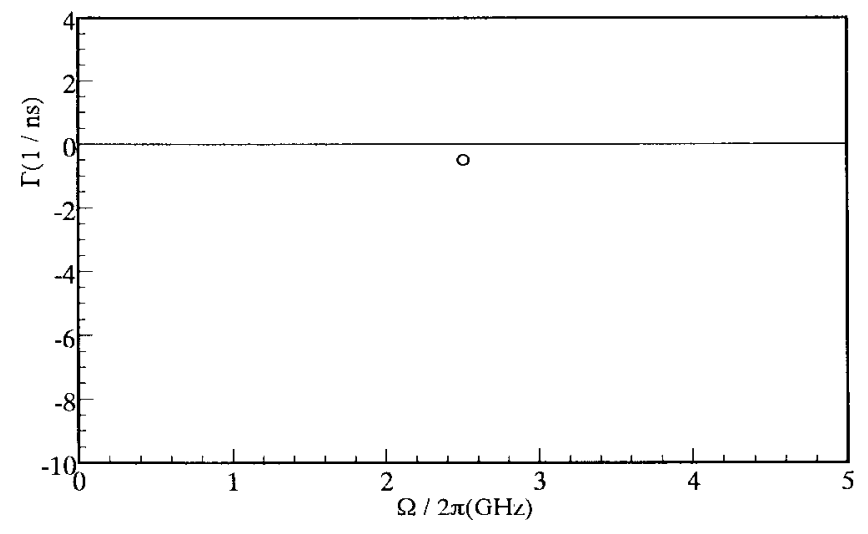

(a)

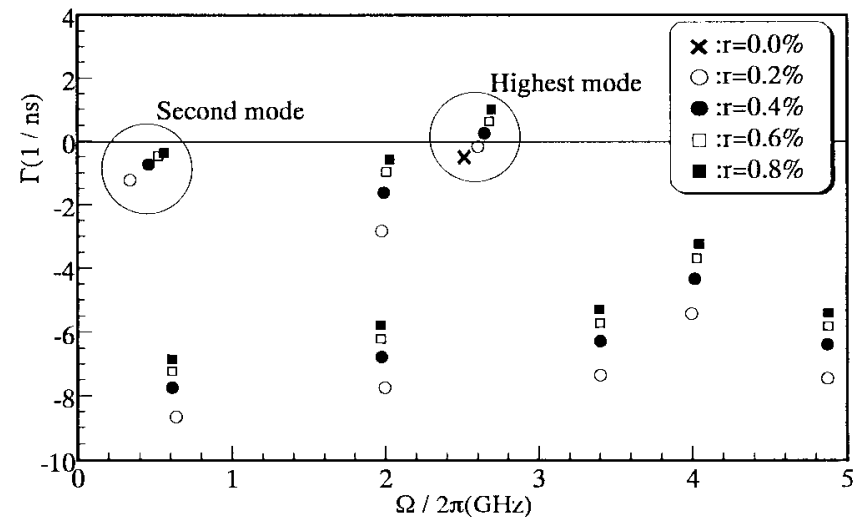

(b)

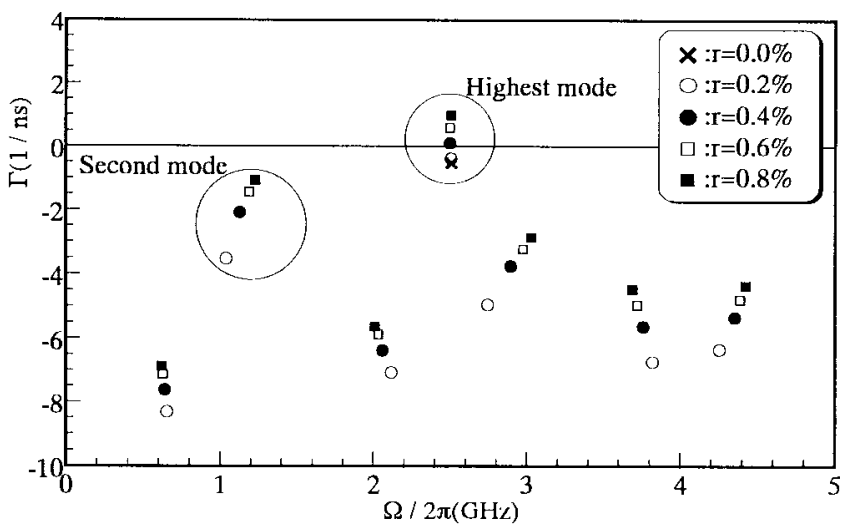

(c)

Fig. 5. Linear mode distributions calculated from (6) and (7) for the increase of $r$ at $J=1.3 J_{\mathrm{th}}$ and $L=10.0 \mathrm{~cm}$. (a) Solitary laser (i.e., without optical feedback), (b) PCF, and (c) CFB.

the value of the real part of the relaxation oscillation mode for the solitary laser is negative, the laser output shows a damping oscillation for this mode. The value of the imaginary part of this mode corresponds to the relaxation oscillation frequency, and it is $f_{R}=2.50 \mathrm{GHz}$ at $J=1.3 J_{\mathrm{th}}$ in this case. On the other hand, there are many linear modes in the presence of optical feedback. Fig. 5(b) and (c) shows the distributions of linear modes for PCF and CFB, respectively. The external amplitude reflectivity is varied from $0 \%$ to $0.8 \%$ for each model. With the increase of the reflectivity, the highest mode, which has the highest value of the real part among linear 
modes, becomes unstable in the first place due to the positive value of the real part. The trend is similar to the case for CFB in Fig. 5(c), but the values of the relaxation oscillation frequencies do not coincide with each other. Furthermore, the modes for the two models are located alternately along the frequency axis. With the increase of the external reflectivity, values of real parts for all modes increase and each mode tends to be less stable. Then the laser output power shows an unstable oscillation with the undamped relaxation oscillation. When the highest mode crosses well over the zero point of the real part, the second mode is excited and the instability is much enhanced. It is also noted that the behaviors of the external cavity mode for PCF [the second mode in Fig. 5(b)] is completely different from that for CFB. The value of the frequency for the second mode for CFB is compatible with that of the frequency calculated from the external cavity length, while that for PCF is much different from it (also compare with Fig. 2).

The laser output power at which the mode crosses the zero point of the real part in the linear mode distribution map shows undamped oscillation (the relaxation oscillation mode). We investigate the stable and unstable boundaries of such oscillations based on the linear stability analysis. Fig. 6(a) shows the change of the relaxation oscillation frequency along the external cavity length. Black circles are the results for PCF and white ones are those for CFB. The relaxation oscillation frequency decreases as the increase of the external cavity length jumps up at the stability peaks and then repeats the same process for the increase of the external cavity length. The results for CFB has already been obtained [2]. The straight line of $f_{R}=3.25 \mathrm{GHz}$ in Fig. 6(a) is the relaxation oscillation frequency for the solitary laser at $J=1.5 J_{\mathrm{th}}$. Fig. 6(b) is the critical reflectivity at which the laser output power gets into period-1 oscillation. The solid curve represents the results for PCF and the dashed curve represents those for CFB. These results are compatible with those in the previous paper [22] based on numerical calculations from the rate equations. The difference of stability peaks between two models has also been discussed in the reference. The origin of the stability peak enhancement as shown in Fig. 6(b) is explained in the next figure.

The transition of the highest mode for the variations of the external reflectivity was shown in Fig. 5; however, this mode switches to the other one depending on the position of the external cavity. The alternation of the highest mode for the change of the external cavity length in PCF is shown in Fig. 7. The positions correspond to those around the third peak in Fig. 6(b). The reflectivity is fixed at $r=0.5 \%$. At first, mode A is the highest one at the external cavity length of $L=11.4$ $\mathrm{cm}$ in Fig. 7(a), and this mode induces almost nonvanishing oscillation. At the external cavity length of $L=11.6 \mathrm{~cm}$ in Fig. 7(b), two modes (modes A and B) are competing and their real parts are negative, so that the relaxation oscillation is not excited in this state and the laser output power stays stable. At a further increase of the external cavity length to $L=11.8 \mathrm{~cm}$, the highest mode switches to mode B from A and this mode also induces nonvanishing oscillation. From these results, the stability enhancement is explained by the competition between

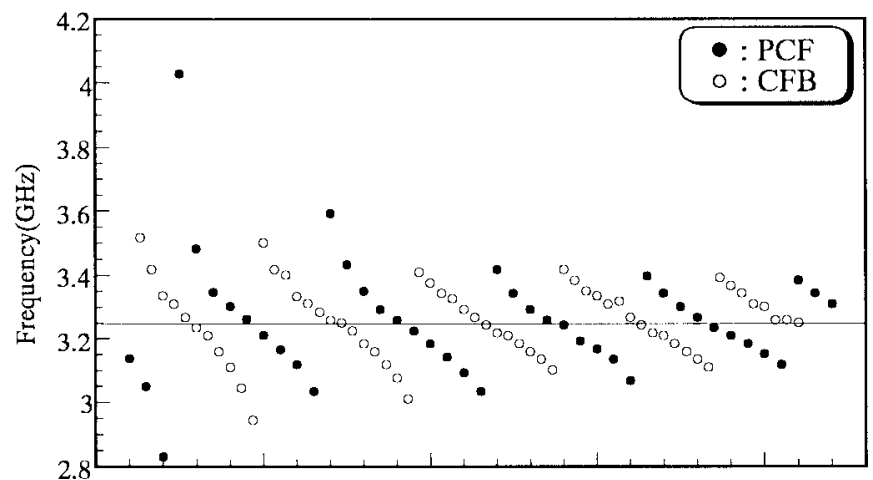

(a)

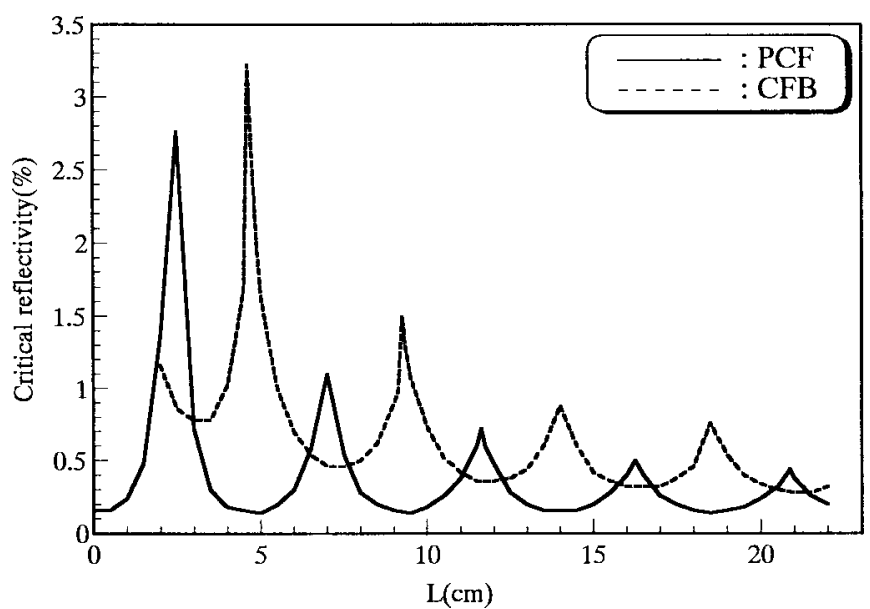

(b)

Fig. 6. (a) Variations of the highest mode frequency for the external cavity length $L$ at which the oscillation becomes nonvanishing state. Black circle: PCF; white circle: CFB. The straight line of $3.25 \mathrm{GHz}$ is the relaxation oscillation frequency of the solitary laser. (b) Boundary between the stable fixed and periodic states for the external cavity length $L$. Solid line: PCF; dashed line: CFB.

the two linear modes near the relaxation oscillation frequency. On the other hand, the laser output power soon evolves into periodic oscillation for a small reflection of light at stability minima such as shown in Fig. 6(b), since only a single mode is always excited.

The frequency of the highest linear mode also changes for the variations of the injection current, and it is shown in Fig. 8. The external cavity length is $L=15.0 \mathrm{~cm}$. The black circle in Fig. 8 represents the frequency for PCF at which the highest mode crosses the zero point of the real part (namely, the laser output power shows a nonvanishing relaxation oscillation), and the white one is that for CFB. The mode frequency has a linear relation with the square root of the injection current, except for frequent jumps. This linearity is consistent with the relation $f_{R}=\sqrt{g E_{s}^{2} / \tau_{p}} / 2 \pi$ for the case of the solitary laser (the power, i.e., the square of the field amplitude, is in proportion to the injection current). With optical feedback, the mode frequency jumps for each frequency separation of $\Delta f=1.0 \mathrm{GHz}$. This frequency separation is the same as that calculated from the external cavity length of $L=15.0 \mathrm{~cm}$. The jump of the frequency is also originated from the alternation of the highest mode for the increase of the injection current. But the jumping positions of the injection currents between PCF 


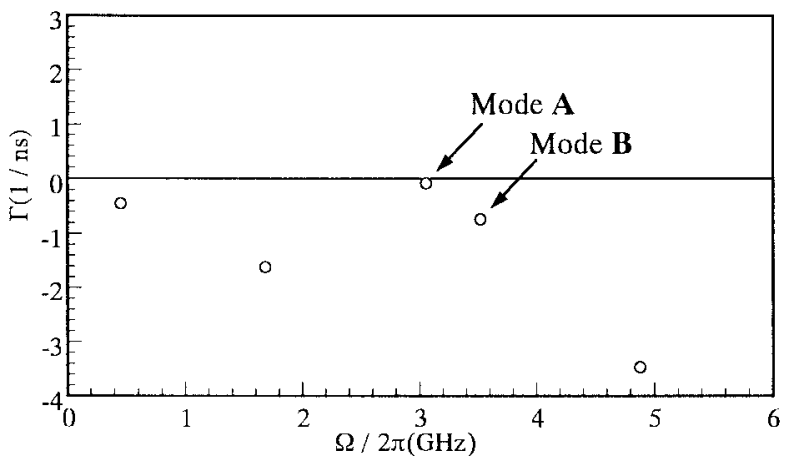

(a)

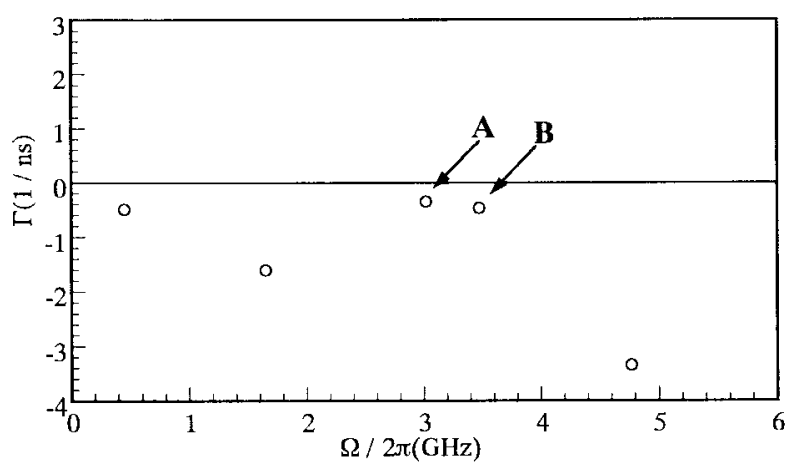

(b)

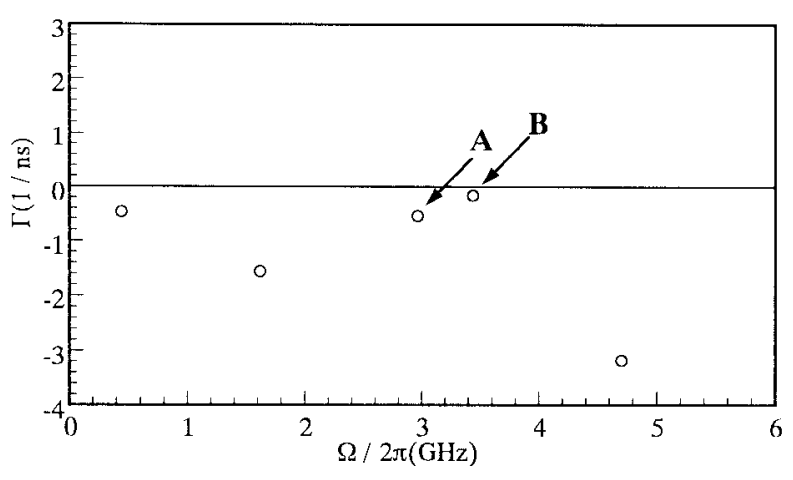

(c)

Fig. 7. Competition of two highest linear modes at $r=0.5 \%$ and $J=1.5 J_{\mathrm{th}}$ for the variations of the external cavity length.

and CFB exist alternately. This is derived from the fact that the linear modes located alternately between the two cases as shown in Fig. 5.

Again, we return to the behavior of the second mode. The black circle in Fig. 3 is the second mode along the external cavity length for PCF. The frequency is calculated from the nonvanishing oscillation frequency of the second mode based on the linear stability analysis. But, strictly speaking, the state already becomes unstable because of the positive value of the real part of the highest linear mode. Therefore, the mode may be not exactly equal to the expected excited second mode frequency for quasi-periodic oscillations of the laser output power. In spite of this fact, the black circle is qualitatively consistent with the actual second mode frequency directly calculated from the rate equations. For comparison, the white circle in Fig. 3 represents the frequency of the second mode

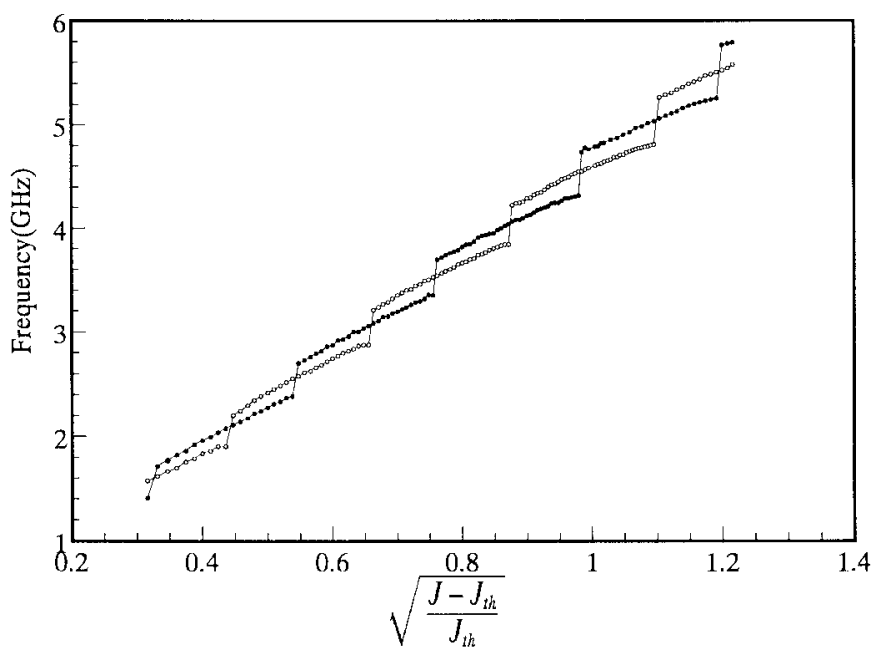

Fig. 8. Variations of the highest mode frequency for the square root of the injection current at which the mode becomes nonvanishing oscillation state. The external cavity length is $L=15.0 \mathrm{~cm}$. Black circle: PCF; white circle: CFB.

for CFB. In this case, the result coincides well with that in the numerical simulation of the rate equations. Thus, the dynamic behaviors not only for the conventional feedback but also for the phase-conjugate feedback are reflected by the distribution and transition of the linear modes with the variations of the parameters.

\section{CONCLusion}

In this paper, we have numerically investigated the dynamics of semiconductor lasers with phase-conjugate feedback and compared the results with those for conventional feedback. In particular, the stability and periodic and quasi-periodic conditions have been studied in detail. The dynamic behaviors have been investigated not only by numerical calculations based on the rate equations but also from linear stability analysis. The reconstruction of the attractors from the time-delayed data has proved to be a very useful tool for examining the dynamics. In the linear stability analysis, we have discussed the route to chaos by excitations of the highest and second mode frequencies. The stability enhancement for the external reflectivity has been interpreted by linear mode competition and alternation of the relaxation oscillation frequencies related to the highest linear modes. To investigate the dynamics, we have only focused on the highest and second modes in the linear stability analysis, but much can be extracted from them for the dynamics of semiconductor lasers with optical feedback. The distribution and transition of the linear modes due to the variations of the parameters reflect the dynamics of the laser output and explain well the dependence of the stability and instability on the system parameters. The difference between the dynamics of the phase-conjugate and conventional optical feedback has also been demonstrated. There exist a lot of experimental studies for conventional optical feedback, but only a few papers have been published for experimental studies of phase-conjugate feedback. Therefore, the experimental study of semiconductor lasers with phase- 
conjugate feedback is expected to compare with theoretical investigations.

\section{REFERENCES}

[1] R. Lang and K. Kobayashi, "External optical feedback effects on semiconductor injection laser properties," IEEE J. Quantum Electron., vol. QE-16, pp. 347-355, 1980.

[2] B. Tromborg, J. H. Osmundsen, and H. Olesen, "Stability analysis for a semiconductor laser in an external cavity," IEEE J. Quantum Electron., vol. QE-20, pp. 1023-1031, 1984.

[3] Y. Liu, N. Kikuchi, and J. Ohtsubo, "Controlling dynamical behavior of a semiconductor laser with external optical feedback," Phys. Rev. E, vol. 51, pp. R2697-2700, 1995.

[4] N. Kikuchi, Y. Liu, and J. Ohtsubo, "Chaos control and noise suppression in external-cavity semiconductor lasers," IEEE J. Quantum Electron., vol. 33, pp. 56-65, 1997.

[5] S. I. Turovels, J. Dellunde, and K. A. Shore, "Nonlinear dynamics of a laser diode subjected to both optical and electronic feedback," J. Opt. Soc. Amer. B, vol. 14, pp. 200-208, 1997.

[6] I. Fischer, O. Hess, W. Elsäßer, and E. Göbel, "High-dimensional chaotic dynamics of an external cavity semiconductor laser," Phys. Rev. Lett., vol. 73, pp. 2188-2191, 1994.

[7] C. Simmendinger and O. Hess, "Controlling delay-induced chaotic behavior of a semiconductor laser with optical feedback," Phys. Lett. A, vol. 17, pp. 97-105, 1996.

[8] Y. Liu and J. Ohtsubo, "Dynamics and chaos stabilization of semiconductor laser with optical feedback from an interferometer," IEEE J. Quantum Electron., vol. 33, pp. 1162-1169, 1997.

[9] J. S. Cohen, R. R. Dentine, and B. H. Verbeek, "The effect of optical feedback on the relaxation oscillation in semiconductor lasers," IEEE J. Quantum Electron., vol. 24, pp. 1989-1995, 1988.

[10] J. Helms and K. Petermann, "A simple analytic expression for the stable operation range of laser diodes with optical feedback," IEEE J. Quantum Electron., vol. 26, pp. 833-836, 1990.

[11] A. M. Levine, G. H. M. van Tartwijk, D. Lenstra, and T. Erneux, "Diode lasers with optical feedback: Stability of the maximum gain mode," Phys. Rev. A, vol. 52, pp. 3436-3439, 1995.

[12] J. Ye, H. Li, and J. G. McInerney, "Period-doubling route to chaos in a semiconductor laser with weak optical feedback," Phys. Rev. A, vol. 47, pp. 2249-2252, 1993.

[13] H. Li, J. Ye, and J. G. McInerney, "Detailed Analysis of coherence collapse in semiconductor lasers," IEEE J. Quantum Electron., vol. 29, pp. 2421-2432, 1993.

[14] A. Ritter and H. Haug, "Theory of laser diode with weak optical feedback. 2. Limit-cycle behavior, quasiperiodicity, frequency locking, and route to chaos," J. Opt. Soc. Amer. B, vol. 10, pp. 145-154, 1993.
[15] M. Münkel, F. Kaiser, and O. Hess, "Spatio-temporal dynamics of multistripe semiconductor lasers with delayed optical feedback," Phys. Lett. A, vol. 21, pp. 68-75, 1996

[16] G. P. Agrawal and J. T. Klaus, "Effect of phase-conjugate feedback on semiconductor laser dynamics," Opt. Lett., vol. 16, pp. 1325-1327, 1991.

[17] G. P. Agrawal and G. R. Gray, "Effect of phase-conjugate feedback on the noise characteristics of semiconductor lasers," Phys. Rev. A, vol. 46, pp. 5890-5898, 1992.

[18] G. R. Gray, D. Huang, and G. P. Agrawal, "Chaotic dynamics of semiconductor lasers with phase-conjugate feedback," Phys. Rev. A, vol. 49, pp. 2096-2105, 1994.

[19] L. N. Langley and K. A. Shore, "Intensity noise and linewidth characteristics of laser diodes with phase conjugate optical feedback," Proc. Inst. Elect. Eng., vol. 141, pt. J, pp. 103-108, 1994.

[20] B. W. Liby and D. Statman, "Controlling the linewidth of a semiconductor laser with photorefractive phase conjugate feedback," IEEE $J$. Quantum Electron., vol. 32, pp. 835-838, 1996

[21] D. H. DeTienne, G. R. Gray, G. P. Agrawal, and D. Lenstra, "Semiconductor laser dynamics for feedback from a finite-penetration-depth phase-conjugate mirror," IEEE J. Quantum Electron., vol. 33, pp. 838-844, 1997.

[22] A. Murakami, J. Ohtsubo, and Y. Liu, "Stability analysis of semiconductor laser with phase-conjugate feedback," IEEE J. Quantum Electron. vol. 33, pp. 1825-1831, 1997

[23] S. Fukuchi, S-Y. Ye, and J. Ohtsubo, "Relaxation oscillation enhancement and coherence collapse in semiconductor lasers with optical feedback," Opt. Commun., submitted for publication.

Atsushi Murakami was born in Aichi, Japan, in 1972. He received the B.S. and M.S. degrees in optoelectronics and mechanical engineering from Shizuoka University, Hamamatsu, Japan, in 1995 and 1997, respectively. Currently, he is working toward the Ph.D. degree in applied electronics at the Graduate School of Electronic Science and Technology, Shizuoka University.

His research interests are nonlinear dynamics in semiconductor lasers with optical feedback.

Mr. Murakami is a member of the Optical Society of America and the Japanese Society of Applied Physics.

Junji Ohtsubo (M'95), for a biography, see p. 1246 of the July 1998 issue of this JOURNAL. 